\title{
Overcoming default categorical bias in spatial memory
}

\author{
Cristina SAMPaio \\ Western Washington University, Bellingham, Washington \\ AND \\ RANXiao Frances Wang \\ University of Illinois at Urbana-Champaign, Urbana, Illinois
}

\begin{abstract}
In the present study, we investigated whether a strong default categorical bias can be overcome in spatial memory by using alternative membership information. In three experiments, we tested location memory in a circular space while providing participants with an alternative categorization. We found that visual presentation of the boundaries of the alternative categories (Experiment 1) did not induce the use of the alternative categories in estimation. In contrast, visual cuing of the alternative category membership of a target (Experiment 2) and unique target feature information associated with each alternative category (Experiment 3 ) successfully led to the use of the alternative categories in estimation. Taken together, the results indicate that default categorical bias in spatial memory can be overcome when appropriate cues are provided. We discuss how these findings expand the category adjustment model (Huttenlocher, Hedges, \& Duncan, 1991) in spatial memory by proposing a retrieval-based category adjustment (RCA) model.
\end{abstract}

One function of spatial categorization is to provide us with information that can be used to infer a target's specific location from memory. Although knowing a target's category helps us estimate its location, it can also lead to systematic errors (e.g., Friedman, 2009; Huttenlocher, Hedges, \& Duncan, 1991; McNamara, 1986; Stevens \& Coupe, 1978). In a typical spatial memory experiment, individuals are presented with a target within a space and then reproduce the target's location from memory. One standard finding is that individuals misplace the target toward the prototypical location of its region, an effect known as the categorical bias. When the space is a geometric figure, individuals tend to divide the space into regions bounded at the horizontal and vertical axes and reproduce the target's location in the direction of the center of its region. The magnitude of the bias increases with the distance of the target to its region's prototypical location (Huttenlocher et al., 1991). The geometric spaces onto which individuals impose the Cartesian quadrants can be circular, elliptical, triangular, square, and pentagonal (Wedell, Fitting, \& Allen, 2007), indicating that there is a natural categorization scheme for these 2-D spaces. The partition of spaces in this manner is thought to be related to the perception of the larger space on which the task space is placed (e.g., a computer monitor) and/or to the body axes.

The default categorization in geometric spaces has resisted a number of manipulations, and the evidence for its alteration is controversial. Huttenlocher, Hedges, Cor- rigan, and Crawford (2004) manipulated the distribution of targets in a circle in an attempt to influence the organization of the space. They used an uneven distribution of locations, such that the targets were clustered along the vertical and horizontal axes of the circle, hence forming alternative categories bounded at the diagonals and with prototypical locations at the vertical and horizontal axes. Their prediction was that if people categorized the stimuli according to the distribution of targets in the space, target localization should be biased toward the vertical and horizontal axes. However, if people continued to use the default categories bounded at the horizontal and vertical axes, the standard pattern of bias would result (i.e., biases toward the diagonals of the circle). They used various manipulations in four experiments, with the strongest one being a category judgment task that forced an explicit classification of targets into the alternative categories bounded at the diagonal lines (Experiments 3 and 4). In this procedure, the circle was marked with the boundaries of the alternative categories (diagonals), and each resulting V-shaped category (top, bottom, right, and left quarters) was labeled with a number for the participants to use to categorize the targets before reproducing their locations from memory. The researchers found that the default spatial categories were used exclusively in location estimation, despite the explicit alternative categorization task. Their research led them to the conclusion that the use of these default categories in estimation is "immutable" (p. 78). 
In contrast to Huttenlocher et al.'s (2004) study, Verbeek and Spetch (2008) studied the effects of visually partitioning a circular space on location memory and showed that both the default categorical bias (i.e., toward the diagonal lines) and an alternative bias (i.e., toward the horizontal and vertical lines) can be achieved in different conditions. More specifically, they found that sectioning lines that are visible during both encoding and retrieval can "attract" the targets. Hence, when the sectioning lines were the diagonals of the circle, the standard default bias was found (locations were remembered toward the diagonal lines), whereas when the sectioning lines were the horizontal and vertical axes, a different bias was found (locations were remembered toward the $y$ - and $x$-axes). They, however, concluded that in their experiments, the participants treated the sectioning lines as landmarks, and not as category boundaries, and thus, these findings appear to be a phenomenon different from the categorical bias.

The only documented evidence for an actual alteration of default spatial bias has come from Simmering and Spencer (2007; see also Spencer \& Hund, 2002), who showed that perceptual cues specifying an alternative organization of a large table can change the default pattern of bias in that space when the cues remain visible throughout each trial. In this research, participants remembered the location of a spaceship on a table containing varying levels of perceptual support for an arbitrary category boundary. The results showed a systematic memory bias based on the arbitrary categories only when perceptual support was provided to organize the space into such categories. However, these experiments used a special procedure; for example, they used a very small number of targets in a limited test space and provided explicit feedback on each trial. Thus, it is not clear whether a strong default spatial bias can be overcome under more general testing conditions, such as those used in Huttenlocher et al. (2004).

Nonetheless, Simmering and Spencer (2007) provided evidence that the presence of perceptual cues may be critical. That is, it is difficult to impose an alternative categorization on a geometric space in the absence of perceptual support, even when individuals are explicitly instructed to do so. In Experiment 1, we examined whether the presence of perceptual cues, which was absent in Huttenlocher et al. (2004), is sufficient to induce alternative spatial categorization in a general testing paradigm.

\section{EXPERIMENT 1}

To examine whether adding perceptual support for an alternative categorization scheme can alter the default spatial categorization and thus bias in a circular space, this experiment explicitly presented the boundaries of the alternative categories at retrieval on each trial. We tested the hypothesis that in a circular space, as in a large table (Simmering \& Spencer, 2007), a visually presented alternative structure of the space can alter the space's default categorization. Biases toward the horizontal and vertical axes in target localization indicate the use of the alternative categories. Biases toward the diagonal axes in target localization indicate the use of the default categories.

\section{Method}

Participants. The participants were 19 undergraduate students from Western Washington University, who received partial credit to fulfill a course requirement.

Materials. The stimuli in this experiment were blue target squares that appeared inside a circle $18.5 \mathrm{~cm}$ in diameter. The circle was divided at the horizontal axis into two halves for the labeling of the angles (see Figure 1). The angles in the top half were designated as positive, starting with the $0^{\circ}$ angle at the rightmost point of the horizontal axis, proceeding counterclockwise, and ending with the $180^{\circ}$ angle at the leftmost point. The angles in the bottom half of the circle were designated as negative, starting with the $0^{\circ}$ angle at the rightmost point of the horizontal axis, proceeding counterclockwise, and ending with the $-180^{\circ}$ angle at the leftmost point. The target locations were randomly selected between $-180^{\circ}$ and $180^{\circ}$, with the targets in a range of $5^{\circ}-15^{\circ}$ away from the default category boundaries (horizontal and vertical axes) or from the alternative category boundaries (diagonal axes) being the test items. ${ }^{1}$ The rest of the target locations were filler items not included in the analysis (see Figure 1 for a schematic illustration of the targets). The distance of each target to the center of the circle was also randomly selected from 1.0 to $8.2 \mathrm{~cm}$. The participants viewed the stimuli on a computer screen at a distance of approximately $50 \mathrm{~cm}$. The background and the circle were white, the border of the circle was blue, and the diagonal lines of the circle marking the boundaries of the alternative categories were red.

Procedure. The instructions were read to the participants in a room with a computer screen showing a circle with diagonals forming four alternative spatial categories (top, bottom, right, and left quarters). The participants were instructed to learn the locations of each target for reproduction from memory. After the instructions had been read, each participant was randomly assigned to a computer.

There were 320 trials, and on each trial the participants saw a circle at the center of the monitor for $2,000 \mathrm{msec}$, and a blue target square appeared inside the blank circle for $250 \mathrm{msec}$. Then both the target and the circle disappeared, and after a delay of 1,500 msec, a circle with two diagonal lines marking the alternative categories reappeared in which the participants were to reproduce the target location. The participants made their responses by moving the mouse cursor to the location at which they thought the target had appeared and clicking the mouse button. After each response, the participants were required to move the mouse above the circle and leave it there until the circle reappeared. Otherwise, the computer showed an error message, and the participants had to move the mouse above the circle and click at that position to get to the next trial. This procedure was implemented to prevent the participants from using the mouse cursor as an external marker between encoding and reproduction.

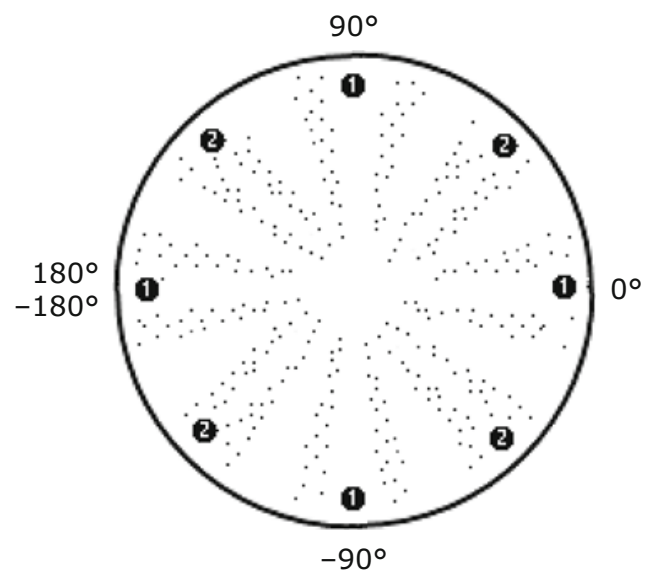

Figure 1. Schematic illustration of target locations and bin assignments. 


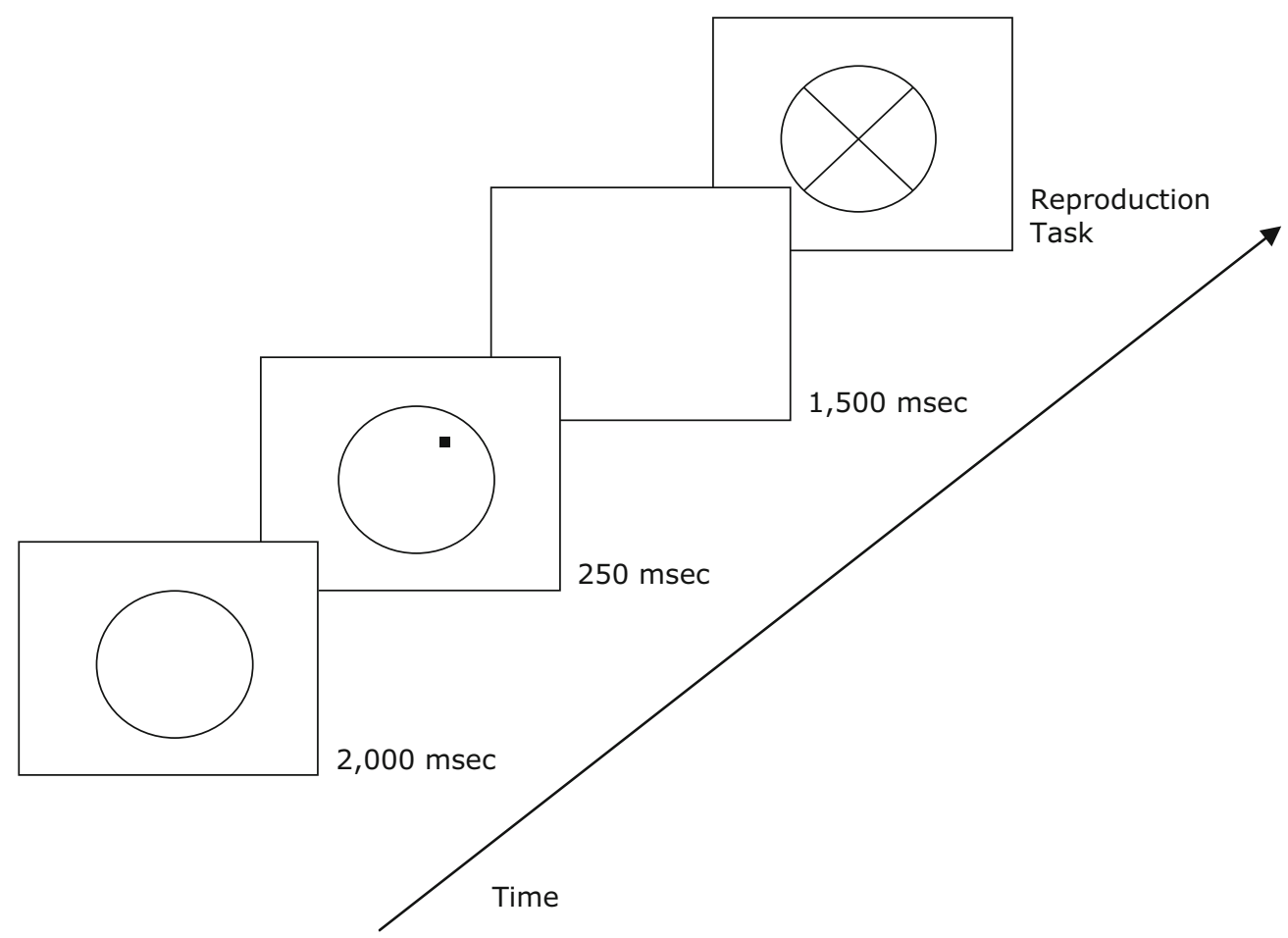

Figure 2. Schematic procedure for Experiment 1.

Figure 2 shows a schematic illustration of the procedure. No feedback was provided on the accuracy of the target localization task.

Data analysis. The use of the default and alternative categories can be observed in the biases of the target localizations, because the bias caused by the use of the default categories is in the opposite direction from that caused by the use of the alternative categories for all the targets. Following the convention (Huttenlocher et al., 1991), the angular bias was calculated as the difference between the reported target angle and the actual angle. We then reversed the sign of the bias for target angles between the ranges $50^{\circ}-60^{\circ}$ and $75^{\circ}-85^{\circ}$ in Quadrant I, ranges $140^{\circ}-150^{\circ}$ and $165^{\circ}-175^{\circ}$ in Quadrant II, ranges $-150^{\circ}$ to $-140^{\circ}$ and $-175^{\circ}$ to $-165^{\circ}$ in Quadrant III, and ranges $-60^{\circ}$ to -50 and $-85^{\circ}$ to -75 in Quadrant IV. Under this coding scheme, bias toward the diagonal lines appeared positive while bias toward the orthogonal lines appeared negative for all targets. Thus, the sign of the bias provides evidence as to what categories are used in estimation, since a positive bias indicates the use of the default categories and a negative bias indicates the use of the alternative categories.

In addition to the sign of the bias providing evidence for what categories are used in estimation, previous research has shown that the effects of a category are strongest on targets near its region's boundary and weakest on targets near its region's center (Huttenlocher et al., 1991). Thus, the bias observed in bins near a category's boundary provides the strongest indication of the influence of that category. If the alternative categories can be used in target localization, it is most likely to be observed in the near-orthogonal targets. Likewise, the effect of the default category should be most obvious in the neardiagonal targets. Hence, to simplify the data analysis, the targets were collapsed into two bins: Bin 1 included targets near the orthogonal axes of the circle, and Bin 2 included targets near the diagonal axes (see Figure 1). The near-orthogonal Bin 1 included angles between the ranges $5^{\circ}-15^{\circ}$ and $75^{\circ}-85^{\circ}$ in Quadrant $\mathrm{I}$, ranges $95^{\circ}-105^{\circ}$ and $165^{\circ}-175^{\circ}$ in Quadrant II, ranges $-105^{\circ}$ to $-95^{\circ}$ and $-175^{\circ}$ to $-165^{\circ}$ in Quadrant III, and ranges $-15^{\circ}$ to $-5^{\circ}$ and $-85^{\circ}$ to $-75^{\circ}$ in Quadrant IV. The near-diagonal Bin 2 included angles between the ranges $30^{\circ}-40^{\circ}$ and $50^{\circ}-60^{\circ}$, ranges $120^{\circ}-130^{\circ}$ and $140^{\circ}-150^{\circ}$, ranges $-120^{\circ}$ to $-130^{\circ}$ and $-150^{\circ}$ to $-140^{\circ}$, and ranges $-40^{\circ}$ to $-30^{\circ}$ and $-60^{\circ}$ to $-50^{\circ}$ in Quadrants I, II, III, and IV, respectively. Figure 1 illustrates this assignment of targets into the two bins.

\section{Results and Discussion}

Responses that were more than $40^{\circ}$ away in either direction from the actual target were considered errors and were excluded from the analysis (3.5\% of the responses). The data from 4 participants were not included in the analysis because they failed to follow instructions.

The first set of analyses examined our main question of whether perceptual support of the alternative categorization (Simmering \& Spencer, 2007) led the participants to overcome the default categorization of the circle. Our results showed that the bias of the near-diagonal targets was not different from zero $[M=.15 ; t(14)=-0.37$, $p=.72]$, but that there was a significant positive bias (i.e., default category effect) for the near-orthogonal targets $[M=.97 ; t(14)=2.92, p<.01]$. Furthermore, the mean angular bias for the near-orthogonal bin was significantly larger than that for the near-diagonal bin $[t(14)=3.42$, $p=.004]$. These results suggest the use of the default category but not the alternative category. Table 1 displays the means and standard deviations.

Overall, the data showed no evidence of the use of the alternative categories in estimation, suggesting that people continued to use the default categories in estimation despite being physically presented with the boundaries of the alternative categories at retrieval on each trial. Our results are inconsistent with those of Simmering and Spencer (2007), in that their research showed that spatial memory depended on the perceptual structure provided in a larger space. We found that presenting diagonal axes 
Table 1

Mean Bias (With Standard Deviations) by Bins for Experiments 1, 2, and 3

\begin{tabular}{|c|c|c|c|c|}
\hline & \multicolumn{2}{|c|}{$\begin{array}{l}\text { Near-Orthogonal } \\
\text { Bin }\end{array}$} & \multicolumn{2}{|c|}{$\begin{array}{c}\text { Near-Diagonal } \\
\text { Bin }\end{array}$} \\
\hline & $M$ & $S D$ & $M$ & $S D$ \\
\hline Experiment 1 & 0.97 & 1.28 & 0.15 & 1.56 \\
\hline Experiment 2 & 0.77 & 2.14 & -3.97 & 3.83 \\
\hline Experiment 3 & -0.18 & 3.55 & -2.01 & 2.83 \\
\hline
\end{tabular}

in a small circular space to mark alternative categories did not change the pattern of the default bias. It should be noted, however, that our method and procedure were different from theirs in a number of ways, such as the scale of the task space, the shape of the space, the number of targets used, the method of locating targets, and the inclusion of accuracy feedback. Any difference or a combination of these differences can account for the contrasting results.

These results are, on the other hand, consistent with Huttenlocher et al.'s (2004) finding that the default category is extremely robust and difficult to overcome in a circular space. We think that there is at least one possibility why the participants in our Experiment 1 and in Huttenlocher et al.'s (2004) study failed to use the alternative categories. According to the category adjustment (CA) model, target locations are encoded at a fine-grain and a coarse-grain (categorical) level of detail, and the category bias results from the blending between these codings. If the participants in our Experiment 1 and in Huttenlocher et al.'s (2004) research initially encoded a target's alternative category membership, instead of the default category membership, their recall of the target would have most likely reflected a bias based on the alternative categorization scheme. However, if the participants did not initially encode the alternative category membership but encoded the default category instead, explicit postencoding presentation of an alternative categorization scheme cannot override the default category coding, because it does not provide any membership information. More specifically, in order to use the alternative category, one must know the target's alternative category membership. To find out a target's alternative category membership, one has to recall its position first, and according to the CA model, such a recall leads to the retrieval of the initially encoded default category representation. In other words, it is possible that target recall using the default category occurred before the alternative category membership was available. It is most likely, therefore, that this recalled target was eventually reported because there was no particular reason to discard the initial recall and regenerate a new one.

Thus, on the basis of the CA model, we hypothesize that (1) the perceptual cues used in our study were not sufficient to induce an initial encoding of the alternative category membership and (2) perceptual cuing without alternative category membership information is ineffective for alternative category use. According to these hypotheses, participants may use an alternative categorization if the alternative category membership of a target (instead of the overall alternative organization of the space) is presented during the retrieval stage, because it eliminates the need to recall the initially encoded default category membership and allows the alternative category information to be integrated with the fine-grain level representation directly. In Experiment 2, we examined this possibility by visually providing each target's alternative category membership during the reproduction period.

\section{EXPERIMENT 2}

\section{Method}

The method was the same as that in Experiment 1, except that the participants were presented with the category membership of each target. Fifteen undergraduate students at the University of Illinois at Urbana-Champaign participated in the experiment and received partial credit to fulfill a course requirement. On each of the 320 trials, the participants saw a circle at the center of the monitor for $2,000 \mathrm{msec}$; then they saw a blue square target appear inside the circle for $250 \mathrm{msec}$. Then both the target and the circle disappeared, and the computer screen turned blank white. A circle reappeared after a 1,000-msec delay, with two diagonal radius lines marking the boundaries of the target's alternative spatial category (i.e., the top, the bottom, the right, or the left quarter of the circle). Figure 3 shows a schematic illustration of the procedure. The participants were told that the region to which the target belonged would be marked to facilitate their target localization. The participants made their response by moving the mouse cursor to the location of the target and pressing the mouse button.

\section{Results and Discussion}

Responses that were more than $40^{\circ}$ away in either direction from the actual target were considered errors and were excluded from the analysis ( $2.5 \%$ of the responses). The results showed a significant negative bias for the neardiagonal targets, suggesting an effect of the alternative category on estimations $[M=-3.97 ; t(14)=4.00, p=$ $.001]$. In contrast, there was no significant bias for the near-orthogonal targets, suggesting no effect of the default category on estimations $[M=0.77 ; t(14)=1.39$, $p=.19]$. The mean angular bias in the near-diagonal bin was significantly larger than the mean angular bias in the near-orthogonal bin $[t(14)=2.26, p=.04]$. These results suggest that the participants used the alternative categories in their target reproduction, instead of the default categories. Table 1 shows all means and standard deviations.

These data contrast with those in Experiment 1 and in previous experiments by Huttenlocher et al. (2004) that failed to alter the use of the default categories in a circular space. These results are also in contrast with those of Simmering and Spencer (2007), who found a stronger bias based on the default category as compared with an induced category in a larger space (a table). The results of Experiment 2 show that people can overcome default categorization in a circular space when alternative categorical membership information is spatially displayed during retrieval.

Another possible explanation for the effect of the category membership is that it provides explicit constraints on the responses that can be made. That is, it encourages individuals to respond inside the diagonal region and thus "truncates" off potential responses outside that region. 


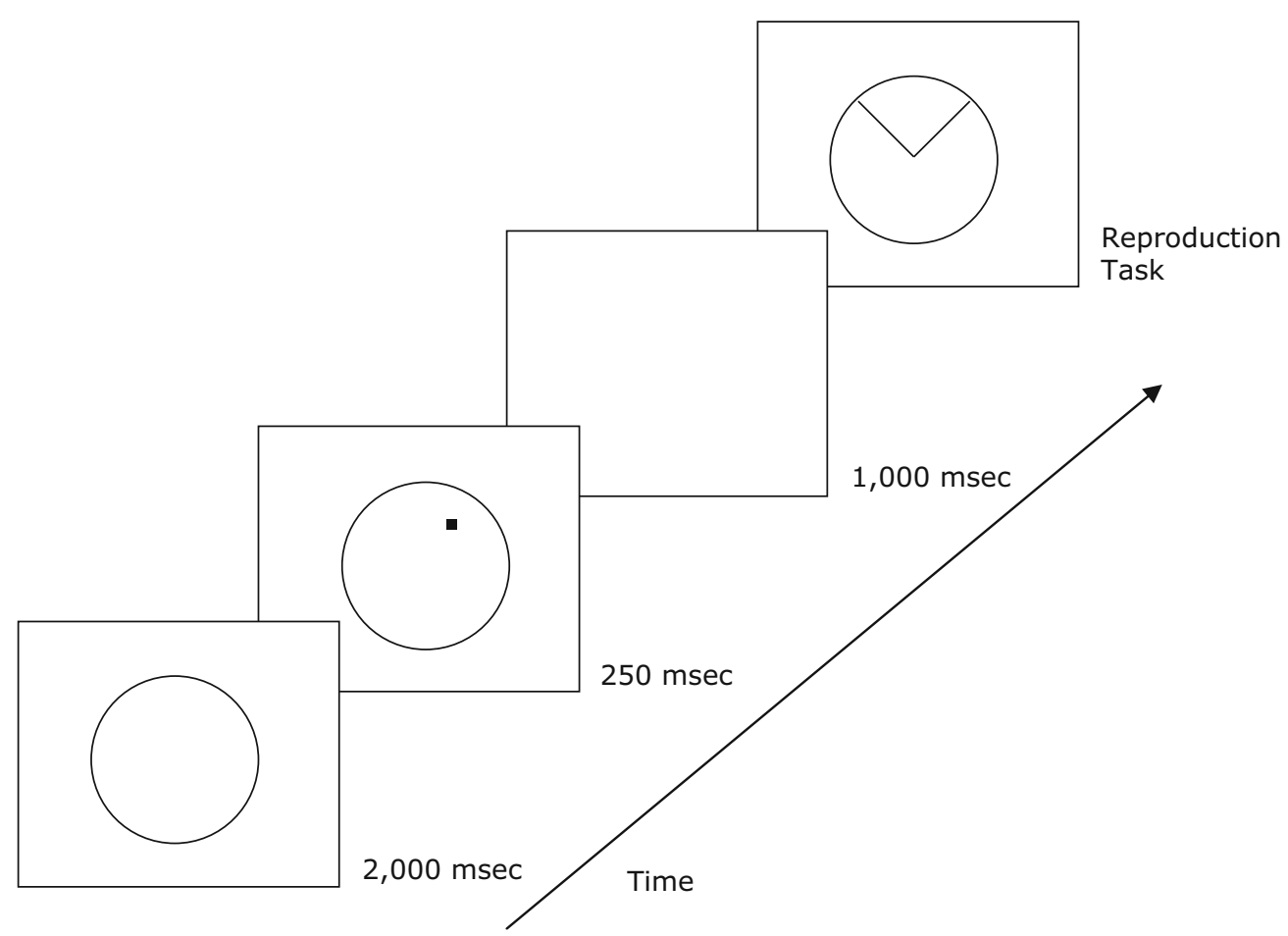

Figure 3. Schematic illustration of the procedure for Experiment 2.

Because this restriction is asymmetrical around the target, more truncation should occur at one side of the distribution than at the other, leading to a bias in the mean responses that mimic the real categorical effect.

To test whether asymmetrical truncation of the response distributions is sufficient to alter the pattern of the default bias (i.e., eliminating the default category bias in the near-orthogonal targets and creating an alternative category bias in the near-diagonal targets), we artificially imposed diagonal boundaries on the data for Experiment 1 and excluded all responses that were outside the target's corresponding alternative category (i.e., responses that would have been truncated by the constraints imposed by its target's alternative category boundaries). In total, 109 trials $(10 \%)$ in the near-diagonal bin were truncated by the imposed boundaries, resulting in a negative bias in the near-diagonal bin $[M=-1.78 ; t(14)=-3.75, p=$ $.002]$. However, none of the trials in the near-orthogonal bin included in the original analysis was excluded by the imposed category boundaries. Thus, the significant positive bias for the near-orthogonal targets remained, indicating that the effect of the default category on estimations was not eliminated by the artificially imposed alternative category boundaries. These data suggest that the truncation hypothesis alone is insufficient to explain the different patterns of bias in the two experiments. These data suggest that the effect of the alternative category membership is more likely due to a combination of the alternative category information with the fine-grain representation of a target location, rather than to the response constraints imposed by the boundaries alone.
Overall, our data in Experiment 2 showed that membership cuing can dominate the use of default categories in location memory. Because this finding is an isolated case among some controversial findings, we conducted Experiment 3, using a different type of cue in an attempt to establish further the alteration of the default categorical bias. In Experiment 3, we investigated whether a nonspatial source of membership information could also be used to overcome the default categorization scheme of the circular space. We chose to use a nonspatial source of membership information on the basis of research that has shown that in addition to spatial information, geographical knowledge contains a variety of other information about a given region, such as its climate, the languages spoken, its time zone, and so forth (Friedman \& Brown, 2000). In Experiment 3, we applied this type of finding to the circular space to test whether a target's nonspatial features can also be utilized to organize the space and overcome the default categorical bias. To test our hypothesis, we provided feature cues to an alternative category membership, as opposed to providing, at the response stage, the default membership based on diving the circle into the Cartesian axes. More specifically, each alternative category was defined by a unique pairing of target shape and color (e.g., black squares were members of one alternative category, and green crosses of another). On the basis of the classic view of categorization that all stimuli that share the defining features of a category are members of that category (Smith \& Medin, 1981), we predicted that the individuals in our experiment would use the defining features of a target to categorize it in space. 


\section{EXPERIMENT 3}

In Experiment 3, we used a unique pairing of shape and color for targets that were members of each alternative category. To reinforce the alternative target categorization, we showed a preview of a sample distribution of targets from all four categories. We hypothesized that if this target-level information was used to form spatial categories, participants would place category boundaries at the diagonals, rather than or in addition to those at the horizontal and vertical axes.

\section{Method}

Participants. The participants were 15 undergraduate students at Western Washington University, who received credit to partially fulfill a course requirement.

Materials. In the preview phase, a blue circle $18.5 \mathrm{~cm}$ in diameter displayed 200 targets of approximately $3.1 \mathrm{~mm}$ each. The targets were randomly generated from $-180^{\circ}$ to $180^{\circ}$, with the constraint that they were at least $5^{\circ}$ away from the diagonal lines. There were no lines marking the boundaries of the alternative categories, but the distribution of the targets indicated four $\mathrm{V}$-shaped regions within the circle (top, bottom, left, and right), each of which was defined by a unique pairing of target shape and color. As Figure 4 illustrates schematically, the top wedge was specified by black squares, the bottom wedge by green crosses, the right wedge by pink triangles, and the left wedge by blue circles.

In the presentation and test phases, the stimuli were the same as those in Experiment 1, except that the targets had the shape and color corresponding to their alternative category membership, instead of being blue squares, and no boundaries were visible at retrieval.

Procedure. The instructions were read to the participants in a room with a printout of the preview screen showing 200 targets from all four alternative categories placed within its corresponding region in a circle. The participants were explicitly told that the stimuli were divided into four categories, each corresponding to targets of a specific pairing of color and shape. For example, green crosses defined the bottom V-shaped category, and black squares defined the top V-shaped category. After the instructions had been read, each participant was randomly assigned to a computer. The experiment started with a preview phase, in which the participants were allowed to look again at the circle with a sample distribution of the targets for as long as they desired. The preview image then disappeared, and the 320 test trials began.

On each trial, the participants saw a blank circle for $2,000 \mathrm{msec}$; then a target appeared within the circle for $250 \mathrm{msec}$. After a 1,500-msec delay, during which a white blank screen was shown, the blank circle reappeared, and the participants were asked to place the mouse at the exact location at which the target had appeared and to click on it.

\section{Results and Discussion}

The data from 1 participant were excluded from the analysis because the participant failed to follow instruc-

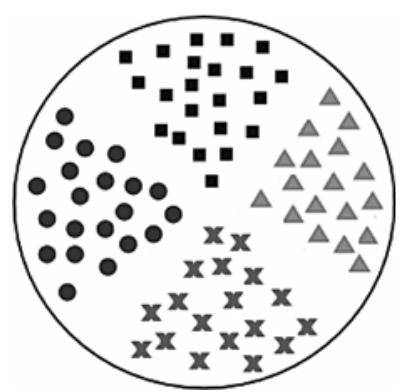

Figure 4. Schematic distribution of targets in Experiment 3. tions. The trimming procedure was the same as that in Experiments 1 and 2 and excluded $6.6 \%$ of the responses.

Our analysis showed that the alternative category was exclusively used in estimates. There was a significant negative bias for the near-diagonal targets $[M=-2.01$; $t(13)=2.67, p=.02]$ and a nonsignificant bias for the near-orthogonal targets $[M=-0.18 ; t(13)=0.19, p=$ $.86]$, suggesting that participants can overcome the default category bias and rather use the alternative category in target reproduction. The mean angular bias in the neardiagonal bin was not significantly larger than that in the near-orthogonal bin $[t(13)=1.60, p=.14]$. These findings replicate those in Experiment 2 and suggest that target feature information, such as shape and color, can be used to form alternative spatial categories within a circle and that these categories are used in the process of location estimation. Table 1 shows all the means and standard deviations.

\section{GENERAL DISCUSSION}

In this article, we have examined whether strong default spatial categorization in a circular space can be overcome and when. Consistent with the findings in Huttenlocher et al. (2004) that default categories are very robust and difficult to overcome, visual presentation of the category boundaries did not induce the use of the alternative category in location estimation, even when provided on each trial. In contrast to Experiment 1, we found that direct visual cuing of the alternative category region the target belonged to during retrieval (Experiment 2) or unique target feature information associated with each alternative category available during encoding (Experiment 3) successfully led to the use of the alternative category.

These findings suggest that default spatial categories are generally very persistent but can be dominated by alternative categorization when appropriate cues are provided. The finding is consistent with that found in spaces without strong default categorization, such as natural environments (e.g., Hirtle \& Jonides, 1985), and in more complex semantic knowledge domains, such as geography (e.g., Friedman \& Brown, 2000). Previous research in these areas has shown that both spatial and nonspatial factors can impact spatial categorization and judgment (e.g., McNamara, Hardy, \& Hirtle, 1989). For example, in a natural environment without a well-defined hierarchical structure, Hirtle and Jonides found that people can use a number of sources of information, such as proximity and semantic knowledge, to categorize the space. Their data showed that participants clustered landmarks in a city on the basis of spatial factors, such as proximity, as well as nonspatial properties, such as frequency of names on route, and that participants then made spatial judgments on the basis of these subjective clusters. In the geography domain, nonspatial sources of information also have been shown to be the basis for spatial judgments, in addition to spatial sources of information. Friedman and Brown, for example, reported that geographical knowledge contains a variety of information about a given region that is not spatial, such as the climate of the region. 
Our interpretation of the present results is close in spirit to Reyna's (1992) fuzzy-trace theory, which postulates that memories for gist and verbatim information are dissociated from each other. According to the fuzzytrace theory, the amount of error in memory varies with the relative accessibility of the gist (categorical) and the verbatim (exact) information. It is assumed that the verbatim memory decays more rapidly than the gist memory and, hence, that responses are likely to be based more on gist than on verbatim information as memory delays increase (e.g., Reyna, 1992). Building on this idea, we think that the type of bias in locating targets in a circular space (toward the prototype of the default or of the alternative categories) is also explained by the relative accessibility or strength of these representations.

The results of Experiment 1 are consistent with the predictions of both a category-induced bias account (e.g., Huttenlocher et al., 1991) and a landmark-based encoding account (Verbeek \& Spetch, 2008). According to the landmark-based encoding account, the participants in Experiment 1 may have treated the dividing diagonal lines as landmarks, rather than as category boundaries, and thus reproduced target locations toward these landmarks, rather than toward the prototypical location of the default categories. However, the data from Experiments 2 and 3, although consistent with the category-based account, cannot be interpreted using a landmark-based encoding account, because we found a bias toward the orthogonal axes and there were no landmarks at these axes to induce a landmark attraction bias. Thus, taken together, our findings are more in line with the category-based account.

Our findings contribute to the literature as it resolves the controversial question on the alteration of strong default categorization scheme and bias. Huttenlocher et al. (2004) varied the distribution of targets to influence the organization of a circular space but found that despite a number of seemingly strong manipulations, people continued to use geometric default categories bounded at the horizontal and vertical axes. The authors concluded that the use of the default categories within a circle was "immutable" (p. 78). In contrast, Simmering and Spencer (2007) showed that visible perceptual cues specifying an alternative organization of a large table can change the default pattern of bias in that space, although a special experimental procedure was used in their studies. Our findings demonstrated that alternative categorization in spaces with strong default categories (such as a circle) can be achieved under at least two conditions - namely, when the cue contains information about an alternative category membership either presented spatially at retrieval (Experiment 2) or specified by a unique pairing of target color and shape (Experiment 3), available at encoding.

The exact conditions under which the alternative categorization can be achieved are still not completely clear. Our results do not suggest that category membership information is necessary for the use of alternative categories. It is possible that other forms of categorical information, when made compelling enough, are effective as well. Nor do our results suggest that category membership information is sufficient. For example, it is unknown whether other types of category membership information (e.g., regionspecific auditory cues presented along with the target, a verbal cue indicating the target region instead of the "V" cue, etc.) will be equally effective. Moreover, it should be noted that we made spatial membership information available only at retrieval in Experiment 2, whereas nonspatial membership information was made available during encoding in Experiment 3. How effective each spatial and nonspatial cue is, when during the memorial process each cue is most effective, and how these various cues may interact with each other are issues that remain to be resolved by future research.

\section{A Retrieval-Based Category Adjustment Model}

Huttenlocher et al. (1991) proposed the CA model to explain the systematic bias observed in object localization. The CA model postulates that (1) object locations are encoded at two levels of detail, a fine-grain (metric) level and a coarse-grain (categorical) level; (2) the category bias results from the blending between these codings; and (3) the weights of each of the codings in the blending are determined by the uncertainty of the fine-grain representation and the category concentration (Huttenlocher, Hedges, \& Vevea, 2000). They further suggested that this hierarchical coding system compensates for the fast-decaying fine-grain representation and can improve overall localization performance, despite the systematic bias. However, the original CA model did not explicitly define the temporal locus of the blending between different levels of codings. A recent study by Sampaio and Wang (2009) showed that the category bias does not result from people's holding a default-category-integrated memory but, rather, that the bias arises from a blending process operating on undistorted memories at the response stage.

The present experiments further suggest that when multiple categorical information is available, a selection and a weighting process occur during the blending stage. We hypothesize that the blending may occur among all information available at that time, and it is possibly based on the strength and reliability of the various codings. In Experiment 1 , the default categories continued to be used, despite visual support of the boundaries of an alternative categorization scheme, because they provided no information about the alternative category membership. In Experiment 2, we provided cues that contained spatial information about alternative category membership at retrieval. Hence, the spatial cue made the alternative category membership more available and reliable than the potential default category coding and, thus, led to the use of the alternative category in estimation. In Experiment 3, we provided nonspatial cues that contained unique information about alternative category membership, and these target feature cues provided reliable information during encoding on the alternative category membership and, thus, led participants to abandon the default categorization scheme. These findings are consistent with the nonspatial memory research, which has shown that individuals use the most available schema to guide their retrieval of specific episodic items even when the items had not originally been encoded in the context of that schema (e.g., Anderson \& Pichert, 1978). 
On the basis of the CA model and these more recent findings, we propose a retrieval-based CA model (RCA model). The assumptions of our proposed RCA model are that (1) the target location can be represented at multiple levels, including a specific coding and one or more categorical codings that can be acquired at any time between encoding and retrieval; (2) these mental representations are retained separately; (3) the representations can become less reliable/accessible over time, with the specific coding showing faster decay than the categorical codings; and (4) during the response stage, the strength/reliability of each representation is evaluated, and the output is a weighted combination of the multiple codings.

Our proposed RCA model can explain the systematic default bias shown originally in Huttenlocher et al. (1991), as well as the successful recognition of the original target position despite biased recall performance shown by Sampaio and Wang (2009). It can also explain findings that the category effect increases over time. With the passage of time, the specific representation decays more and more in comparison with the categorical representation. Consequently, over time, there is a greater reliance on the categorical coding than on the specific coding, even if the specific coding itself remains unbiased throughout the delay, leading to larger biases. Moreover, this model also provides a potential explanation for why the cues used in the present experiments could alter the default categorical bias, whereas the target-distribution manipulation in Huttenlocher et al. (2004) failed. The cues used in Experiments 2 and 3 provided reliable information about which category the target belonged to, whereas the distribution of targets did not. Therefore, the alternative category information was used during the blending process in the present Experiments 2 and 3, but not in Huttenlocher et al. (2004).

Nonetheless, further investigation is needed to test the assumptions of the RCA model. For example, the model predicts that manipulations of the validity of the cues should affect whether they are used in the localization task. Moreover, the RCA model predicts that memory delay should have a differential effect on the size of the biases caused by categorical information acquired during encoding and by categorical information provided at retrieval, because the relative accessibility and reliability of these two types of information can change with the delay duration. In ongoing research, these hypotheses are being investigated.

Overall, we have shown that the default spatial category bias can be overcome in spaces with strong default categorization. These findings are consistent with a retrievalbased CA model.

\section{AUTHOR NOTE}

Experiment 2 reported in this article was part of C.S.'s doctoral dissertation at the University of Illinois at Urbana-Champaign and was sup- ported by NSF Grant BCS 03-17681 to R.F.W. We thank Kelly Merriman and Michelle Wright for their help collecting the data and the anonymous reviewers for their helpful comments. Correspondence concerning this article should be addressed to C. Sampaio, Department of Psychology, Western Washington University, 516 High Street, Bellingham, WA 98225 (e-mail: cristina.sampaio@wwu.edu).

\section{REFERENCES}

Anderson, R. C., \& Pichert, J. W. (1978). Recall of previously unrecallable information following a shift in perspective. Journal of Verbal Learning \& Verbal Behavior, 17, 1-12.

Friedman, A. (2009). The role of categories and spatial cuing in globalscale location estimates. Journal of Experimental Psychology: Learning, Memory, \& Cognition, 35, 94-112.

Friedman, A., \& Brown, N. R. (2000). Reasoning about geography. Journal of Experimental Psychology: General, 129, 193-219.

Hirtle, S. C., \& Jonides, J. (1985). Evidence of hierarchies in cognitive maps. Memory \& Cognition, 13, 208-217.

Huttenlocher, J., Hedges, L. V., Corrigan, B., \& Crawford, L. E. (2004). Spatial categories and the estimation of location. Cognition, 93, 75-97.

Huttenlocher, J., Hedges, L. V., \& Duncan, S. (1991). Categories and particulars: Prototype effects in estimating spatial location. Psychological Review, 98, 352-376.

Huttenlocher, J., Hedges, L. V., \& Vevea, J. L. (2000). Why do categories affect stimulus judgment? Journal of Experimental Psychology: General, 129, 220-241.

McNamara, T. P. (1986). Mental representations of spatial relations. Cognitive Psychology, 18, 87-121.

McNamara, T. P., Hardy, J. K., \& Hirtle, S. C. (1989). Subjective hierarchies in spatial memory. Journal of Experimental Psychology: Learning, Memory, \& Cognition, 15, 211-227.

REYNA, V. F. (1992). Reasoning, remembering, and their relationship: Social, cognitive, and developmental issues. In M. L. Howe, C. J. Brainerd, \& V. F. Reyna (Eds.), Development of long-term retention (pp. 103-127). New York: Springer.

SAmPaio, C., \& WANG, R. F. (2009). Category-based errors and the accessibility of unbiased spatial memories: A retrieval model. Journal of Experimental Psychology: Learning, Memory, \& Cognition, 35 1331-1337.

Simmering, V. R., \& Spencer, J. P. (2007). Carving up space at imaginary joints: Can people mentally impose arbitrary spatial category boundaries? Journal of Experimental Psychology: Human Perception \& Performance, 33, 871-894.

Smith, E. E., \& Medin, D. L. (1981). Categories and concepts. Cambridge, MA: Harvard University Press.

Spencer, J. P., \& Hund, A. M. (2002). Prototypes and particulars: Geometric and experience-dependent spatial categories. Journal of Experimental Psychology: General, 131, 16-37.

Stevens, A., \& Coupe, P. (1978). Distortions in judged spatial relations. Cognitive Psychology, 10, 422-437.

VerbeeK, E., \& Spetch, M. (2008). Distortions in location memory. Psychonomic Bulletin \& Review, 15, 328-336.

Wedell, D. H., Fitting, S., \& Allen, G. L. (2007). Shape effects on memory for location. Psychonomic Bulletin \& Review, 14, 681-686.

\section{NOTE}

1. These regions were chosen because they are most informative about the effects of the two categories.

(Manuscript received April 2, 2009; revision accepted for publication May 11, 2010.) 